\title{
The Essence of Clinical Practice Guidelines for Ossification of Spinal Ligaments, 2019: 4. Treatment of Cervical OPLL
}

\author{
Toshitaka Yoshii \\ Department of Orthopaedic and Spine Surgery, Tokyo Medical and Dental University Hospital, Tokyo, Japan
}

\author{
Keywords: \\ OPLL, Guideline, Cervical spine
}

This article is the fourth part of the seven-article series, The Essence of Clinical Practice Guidelines for Ossification of Spinal Ligaments, published in the Spine Surgery and Related Research, Special Issue: Volume 5, Issue 5.

\section{Surgical Treatment of OPLL}

\section{Summary}

Anterior decompression and fusion is proposed for patients with a high ossification occupancy rate within the spinal canal and patients with kyphosis. However, since the incidence of complications and frequency of reoperation are high with the anterior approach, due care should be paid when selecting the surgical procedure suitable for each individual patient (Recommendation level, 2; evidence strength, C).

\section{Commentary}

No prospective RCTs have compared anterior decompression and fusion and laminoplasty. In a prospective nonrandomized study comparing the two procedures ${ }^{1)}$, in patients with a high ossification occupancy rate within the spinal canal $(\geq 50 \%)$ and patients with kyphosis, the outcomes were better with anterior decompression and fusion compared with laminoplasty. Furthermore, a systematic review comparing the two procedures showed similar results ${ }^{2}$. According to a meta-analysis conducted when preparing this guideline $\mathrm{e}^{3)}$, neurological symptoms showed better improvement following anterior decompression and fusion than following laminoplasty. Particularly, in patients with a high ossification occupancy rate within the spinal canal $(\geq 60 \%)$ and patients with kyphosis, the improvement rate in JOA scores was significantly higher with anterior decompression and fusion than with laminoplasty. Furthermore, with regard to cervical spine alignment, the lordotic angle (C2-C7 angle) was greater following anterior decompression and fusion than laminoplasty. In contrast, excluding neck pain, the incidence of complications was significantly higher with anterior decompression and fusion than with laminoplasty. The incidence of neurological complications, including segmental motor paralysis such as upper limb paralysis, tended to be higher in laminoplasty; however, no significant difference was observed. The rate of reoperation was significantly higher with anterior decompression and fusion than with laminoplasty. Therefore, compared with laminoplasty, anterior decompression and fusion can be expected to result in better improvement in neurological symptoms, particularly in patients with a high ossification occupancy rate and patients with kyphosis. However, the incidences of complications and revision surgery are higher with anterior decompression and fusion than with laminoplasty.

The level of recommendation for this CQ was determined based on these meta-analysis results. This meta-analysis demonstrated that direct decompression by the anterior approach is more useful in improving neurological symptoms than indirect decompression by the posterior approach. In contrast, studies comparing the two surgical procedures were generally retrospective observational studies, and the fact that studies with a high level of evidence were lacking, the balance between benefit and harm in the anterior approach and patient values and preferences was uncertain, and the medical costs involved when performing the anterior approach should be considered. As a result, anterior decom-

Corresponding author: Toshitaka Yoshii, yoshii.orth@tmd.ac.jp

Received: May 10, 2021, Accepted: July 8, 2021

Copyright (C) 2021 The Japanese Society for Spine Surgery and Related Research 
pression and fusion is weakly recommended (suggested) in patients with OPLL with a high ossification occupancy rate and patients with kyphosis.

Conflicts of Interest: The author declares that there are no relevant conflicts of interest.

This is a part of The Essence of Clinical Practice Guidelines for Ossification of Spinal Ligaments (2019), which is listed below.

1. Epidemiology of OPLL, written by Tomohiko Hasegawa, MD, PhD, Hamamatsu University, School of Medicine, Japan. https://doi.org/10.22603/ssrr.20210096

2. Pathology of OPLL, written by Takashi Kaito, MD, $\mathrm{PhD}$, Osaka University Graduate School of Medicine, Japan. https://doi.org/10.22603/ssrr.2021-0074

3. Diagnosis of OPLL, written by Hirotaka Chikuda, MD, $\mathrm{PhD}$, Gumma University, School of Medicine, Japan. https://doi.org/10.22603/ssrr.2021-0118

4. Treatment of Cervical OPLL, written by Toshitaka Yoshii, MD, PhD, Tokyo Medical and Dental University Hospital, Japan. https://doi.org/10.22603/ssrr.20210100

5. Treatment of Thoracic OPLL, written by Shiro Imagama, MD, PhD, Nagoya University Graduate School of Medicine, Japan. https://doi.org/10.22603/ssrr.20210095

6. Diagnosis of OLF, written by Masao Koda, MD, PhD, University of Tsukuba, Japan. https://doi.org/10.22603/ ssrr.2021-0116

7. Treatment of Thoracic OLF, written by Kanji Mori, MD, PhD, Shiga University of Medical Science, Japan. https://doi.org/10.22603/ssrr.2021-0094

The original version of this clinical practice guidelines appeared in Japanese as Sekichu Jintai Kokkashou Shinryo Guidelines 2019, published by the Japanese Orthopaedic Association and the Japanese Society for Spine Surgery and Related Research, and its translated version in English appeared in the Journal of Orthopaedic Science 26 (2021) 145.

\section{References}

1. Sakai K, Okawa A, Takahashi M, et al. Five-year follow-up evaluation of surgical treatment for cervical myelopathy caused by ossification of the posterior longitudinal ligament: a prospective comparative study of anterior decompression and fusion with floating method versus laminoplasty. Spine. 2012;37(5):367-76.

2. Chen Z, Liu B, Dong J, et al. Comparison of anterior corpectomy and fusion versus laminoplasty for the treatment of cervical ossification of posterior longitudinal ligament: a meta-analysis. Neurosurg Focus. 2016;40(6):E8.

3. Yoshii T, Egawa S, Hirai T, et al. A systematic review and metaanalysis comparing anterior decompression with fusion and posterior laminoplasty for cervical ossification of the posterior longitudinal ligament. J Orthop Sci. 2020;25(1):58-65.

Spine Surgery and Related Research is an Open Access journal distributed under the Creative Commons Attribution-NonCommercial-NoDerivatives 4.0 International License. To view the details of this license, please visit (https://creativeco mmons.org/licenses/by-nc-nd/4.0/). 